\title{
Madagascar, l'lle rouge, en une quinzaine d'instantanés
}

\section{Jean Martin}

Photos de Maya Majzoub
A quatre en famille, nous avons passé en octobre dernier trois semaines à Madagascar, appelée aussi Grande Terre (par certains «île-continent»), d'où je rapporte impressions et sensations. Beau pays aux multiples facettes - où la réalité est éloignée du film à succès du même nom qu'aiment nos enfants. Des populations aimables, travailleuses, et pauvres.

Rouge. Les couleurs ou d'autres caractéristiques que l'on donne à quelque chose n'ont parfois qu'un rapport assez lâche avec ce dont il s'agit. Il en va tout différemment à Madagascar, qui frappe comme le pays rouge. Dans la partie centrale des hauts plateaux, très fertile (magnifiques carottes, haricots, manioc, fruits aux étals des marchés), la terre est de couleur brique appuyée, égayée par les grandes taches vert mousse des rizières en terrasses. Les maisons sont de briques de terre cuite et les villages sont remarquables d'homogénéité (les fours artisanaux à cuire la brique sont omniprésents dans certaines régions, ainsi que les fours à charbon de bois). Il y a aussi les tsingy rouges, pas loin de Diego Suarez (Nord), extraordinaires dentelles de calcaires créées par l'érosion.

Baobabs. L'arbre emblématique bien sûr. On n'en voit pas partout mais ils marquent le paysage quand ils sont présents: belles hautes sentinelles au tronc droit, parfois bien ventrus (un de 3 mètres de diamètre sur notre parcours), avec un chapeau de branches plutôt grêles. Avons beaucoup apprécié aussi les pachypodiums, qu'on dit (inexactement) baobabs nains: font penser aux bonsaï, avec des branches sortant d'un tronc court, sorte de panse joufflue sur le sol qui sert de réserve d'eau; nous y étions au moment de la sortie de leurs belles fleurs jaunes.

Lémuriens: Une autre des signatures de l'île, où existent une trentaine d'espèces de ces primates arboricoles cousins des singes et de l'homme: la plupart diurnes et végétariens - feuilles, fruits -, d'autres nocturnes et aussi insectivores. S'ils se sont maintenus à Madagascar et pas ailleurs, c'est qu'ils n'y ont

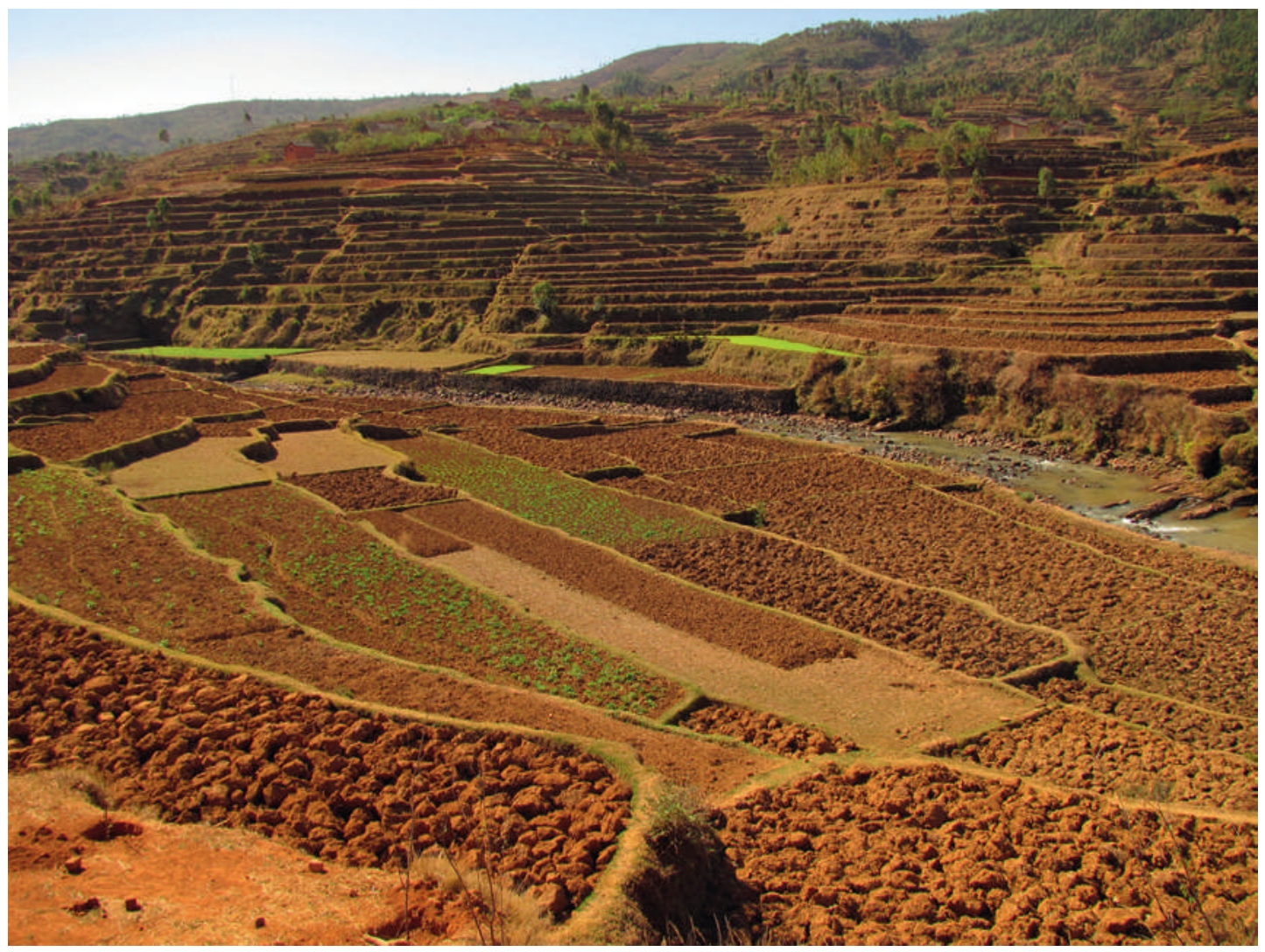




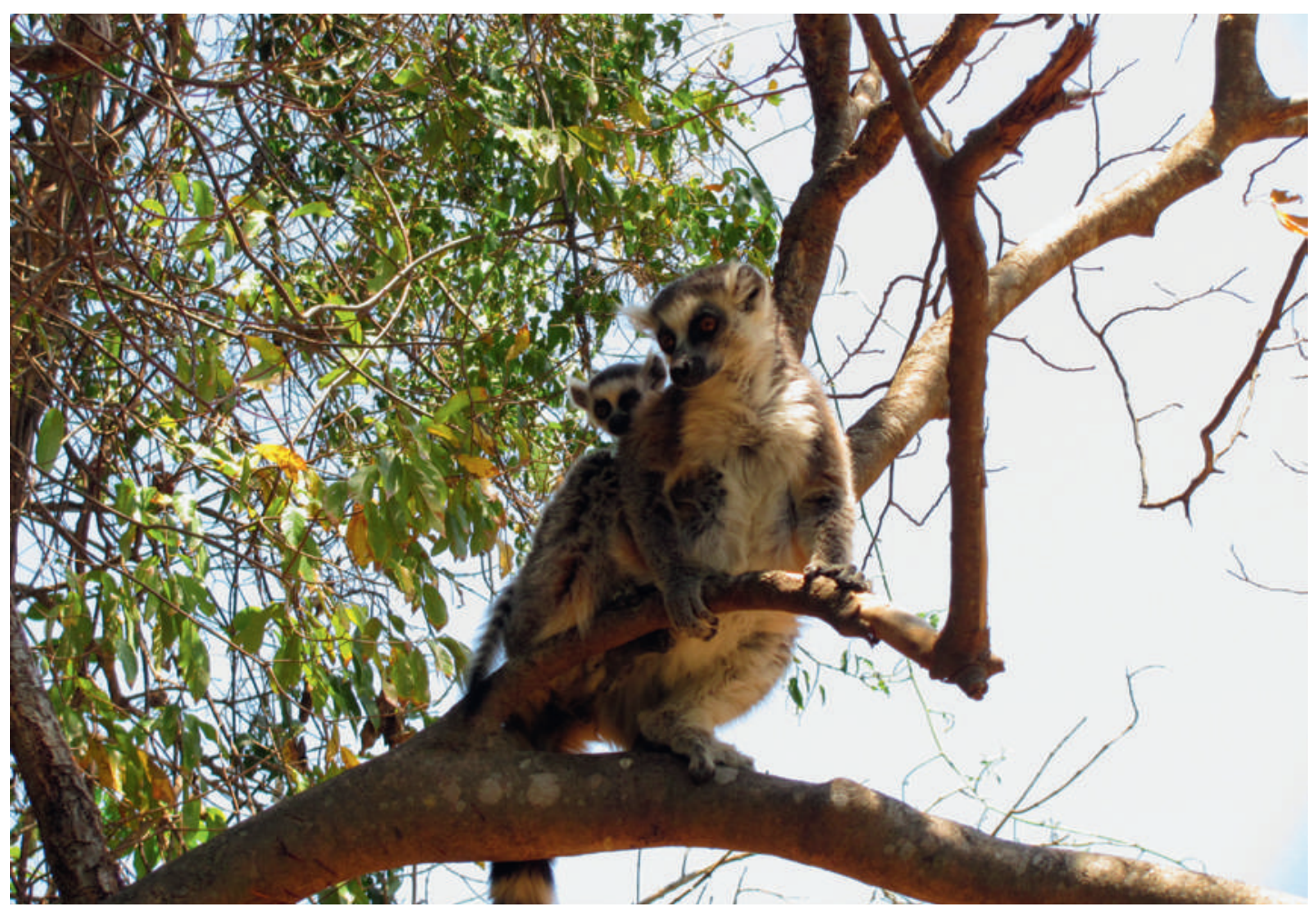

Les lémuriens, emblématiques de Madagascar, aimables habitants des frondaisons.

que peu ou pas de prédateurs - même si le fosa, félidé de la taille d'un chien, en est un. Ils sont néanmoins, comme beaucoup d'autres ailleurs, menacés par l'avancée des activités humaines. Dans les grands et beaux parcs nationaux forestiers comme ceux de Ranomafana et de Ankàrana, on les voit facilement. L'agréable, c'est que quand un rabatteur les a détectés et l'annonce par téléphone portable à votre guide, on a tout le temps de se rendre à l'endroit voulu: les lémuriens continuent tranquillement à manger, làhaut dans les arbres. Tout en montrant comment ils savent sauter de branche en branche, y compris les mères avec leur petit sur le dos.

A mentionner aussi les nombreuses espèces de caméléons de tailles diverses, eux aussi diurnes ou nocturnes, insectivores (plus de la moitié des espèces dans le monde se rencontrent sur la Grande Ile). Ils sont fady (cf. ci-dessous) à de nombreux endroits, parce que réincarnation des Ancêtres.

Ethnies et religions. Tous les guides (livres et personnes) le mentionnent d'emblée, Madagascar est constitué de 18 ethnies principales (plus quelques sous-ethnies), qui gardent manifestement une grande importance (un peu comme les cantons suisses). On estime que le pays a été peuplé à l'époque par des groupes venus d'Indonésie et Malaysie, arrivés sur de frêles esquifs... c'est grand, l'Océan indien! Certains d'entre eux probablement ont caboté par l'Inde, la péninsule arabique, les côtes de l'Afrique orientale. Dans le Centre notamment, le fait est qu'on rencontre des personnes d'allure indonésienne. Sur les côtes, surtout du côté du Canal du Mozambique, l'apport africain apparaît important.
Ces éléments ethniques ont leur reflet dans les coutumes et les pratiques religieuses; les 5\% de musulmans sont concentrés dans le Nord-Ouest de l'île (Diego Suarez, Majunga), qui a des contacts de longue date avec les Iles Comores et Zanzibar proches - on y retrouve aussi les boutres typiques de la côte orientale de l'Afrique.

On considère que la moitié environ de la population est chrétienne (se partageant entre catholiques et protestants, y compris des mouvements évangéliques, certains d'origine africaine). Beaucoup d'églises, en ville et en campagne, pleines lors des services dominicaux.

Animisme. Il y a dans le pays un fond animiste marqué, qui peut être combiné avec les pratiques chrétiennes. Dans diverses circonstances de la vie familiale et communautaire, on sacrifie un zébu (cf. ci-dessous). Nous avons vu sur une île un peu à l'écart mais fréquentée par des touristes un groupe sacrifiant un de ces animaux, dans le but de s'acquérir la bienveillance des esprits et forces naturelles du lieu avant la mise en place d'un développement écotouristique.

Une personne rencontrée disait qu'un touriste s'était perdu en forêt dans un parc national et que, malgré les efforts entrepris, il n'avait pas été retrouvé après une semaine, ajoutant: «C'est la deuxième fois qu'une personne disparaît mystérieusement, il paraît qu'il y a un esprit maléfique.»

Mort, retournement des morts, tombeaux. A Madagascar, les morts sont toujours vivants, ailleurs, et il s'agit d'avoir avec eux les meilleurs rapports. Le décès d'un proche n'est pas autant que chez nous, nous 
dit-on, une occasion de grande tristesse et déchirement, c'est la suite normale et attendue de la vie, qui se poursuit sous une autre forme. Les membres décédés de la famille gardent une influence majeure sur l'existence quotidienne des vivants.

Est pratiqué ce qui est dénommé «retournement des morts», selon des modalités variables suivant les ethnies. D'abord, le cadavre est mis dans une tombe temporaire, durant quelques années. Puis, à un moment déterminé par un songe d'un membre de la famille ou par un astrologue, on se rend à cette tombe où le retournement consiste en un remplacement du linceul original par un nouveau et par la mise dans le tombeau définitif (qui peut être familial). Dans le pays bara (région du Parc national de l'Isalo), les tombeaux définitifs sont souvent des grottes dans les falaises, fermés par des murs de pierres sèches. Ailleurs on voit dans la campagne d'importantes constructions carrées, comme un enclos de jardin, surmontés de totems animistes en bois ou d'une croix. Ces cérémonies sont l'occasion de réjouissances familiales de plusieurs jours.

Circoncision: Arrivant de l'Europe de la bioéthique et du droit, bousculée durant l'été 2012 par les controverses sur la circoncision, j'ai été frappé à Antananarivo de voir la plaque d'un médecin annonçant sa spécialité, la pédiatrie, et sa pratique de la «circoncision selon la méthode américaine»... Je me suis enquis de la place de cette pratique dans le pays: sans que cela ait un caractère religieux, tous les garçons malgaches sont circoncis, de routine; personne ne semble poser la question de l'indication à le faire.

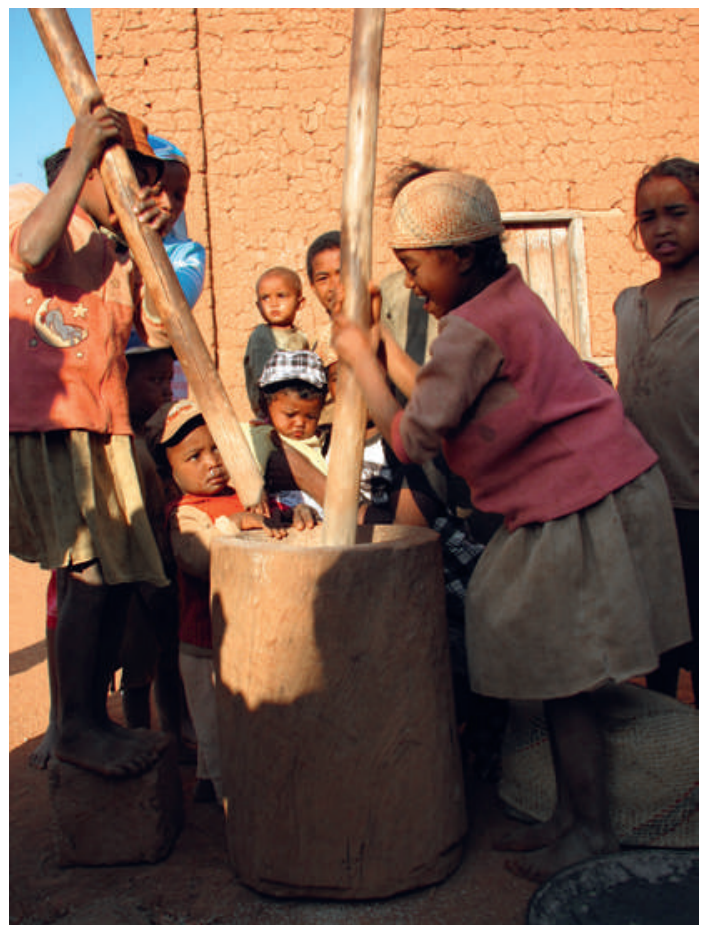

Dans la région betsileo des Hautes Terres, des enfants pilent du maïs.
D'où la surprise de nos interlocuteurs quand j'évoquais l'émoi actuel à ce sujet chez nous.

Les pratiques varient d'une ethnie à l'autre. Dans certaines régions, par exemple chez les Tanala, gens des forêts du Centre-Est, la circoncision est une importante cérémonie communautaire, à intervalle de quatre ou cinq ans, où tout le groupe des garçons d'une classe d'âge est circoncis à la fois. Il n'y a pas de personne spécialement formée chargée de le faire, cela peut-être n'importe quel homme adulte.

Fady (prononcer fad). Ce sont ce qu'en Polynésie (et maintenant partout) on appelle tabou, interdit. Ils sont très divers - et nombreux, la vie sociale malgache en est marquée. Ils peuvent être communs à une assez grande région ou plus souvent être de portée locale, concerner un ou quelques villages voire une famille. Interdictions de certains actes liés à la nourriture, à des moments (jours de la semaine), des chemins qu'on emprunte etc. etc. Même si beaucoup de Malgaches ont reçu une éducation de type moderne, on a l'impression que ces règles sont encore très présentes. Attachement soft à des traditions ou craintes profondément ancrées? Je ne saurais en juger.

Moura moura. Expression survenant souvent dans la conversation, équivalent national de ces formules qui dans de multiples cultures signifient qu'il n'y pas d'urgence, qu'à chaque jour suffit sa peine, qu'il n'y a pas le feu au lac, qu'on verra demain - le mañana latino-américain et ses homologues ailleurs.

Pierres précieuses et villes-champignons. Le sous-sol est riche en pierres précieuses. On y a vécu récemment encore des afflux massifs de population; par exemple la ville de Ilakaka, dans le centre du pays, est surgie très vite quand on y a trouvé en 1998 des saphirs, et compte plusieurs dizaines de milliers d'habitants. Ambiance Far West ou Klondike, pionniers à la recherche de la fortune, échoppes, bars, plaisirs. Mais bien peu sont devenus riches. La traversant, on est frappé de voir aux côtés de beaucoup de maisons misérables les spacieuses villas modernes de ceux qui achètent des pierres précieuses (souvent d'origine indo-pakistanaise) et qui eux s'en tirent bien. Et il n'y pas que les pierres qui fascinent; ailleurs, on voit des orpailleurs dans le lit des rivières.

Téléphone portable et ses dérivés. Se déplacer aujourd'hui dans les pays du Sud fait constater l'omniprésence du portable. Madagascar est pauvre mais, on nous le confirme, «tout le monde» s'est mis à cette technologie (comme d'ailleurs à la télévision). Les enseignes colorées des compagnies du domaine sont omniprésentes. Extension à cet égard, la possibilité de plus en plus utilisée de faire un dépôt d'argent «sur» son téléphone, pour ensuite faire des transferts ou s'acquitter de son dû dans les commerces. J'ai parfois mes doutes sur les bienfaits dans ces pays de certains pans de nos «merveilles» modernes mais là on rend service: très pratique, susceptible d'éviter vols et autres malversations, «léger», écologique même. 


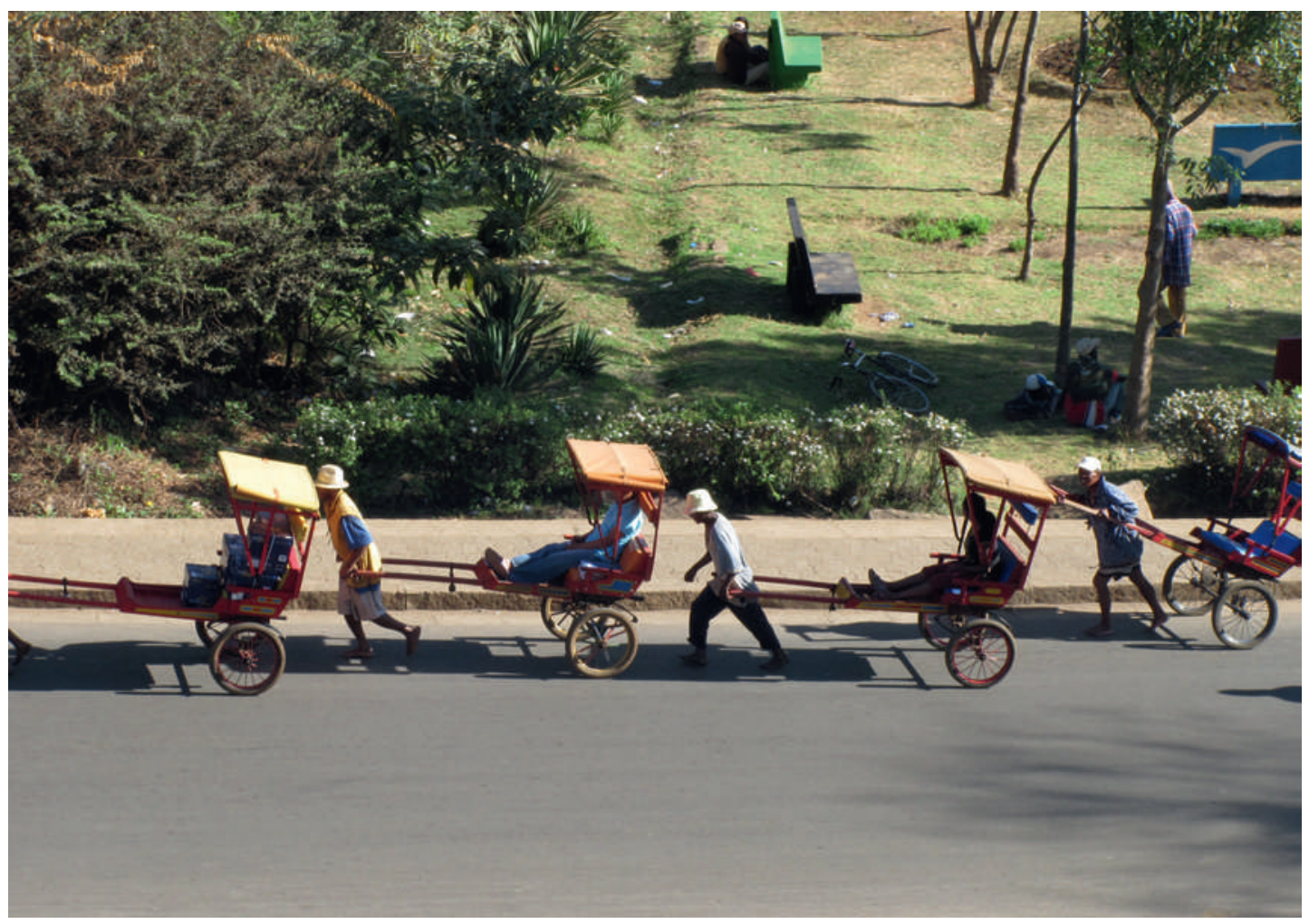

Les pousse-pousse, importés d'Inde par des missionnaires au XIXe siècle, sont très présents. Pour transporter des personnes, comme à Antsirabe et Toliara, ou des marchandises.

Relevons le développement à Madagascar du micro-crédit qui, dans la foulée des travaux de l'économiste et Prix Nobel Muhammad Yunus, met à disposition de (très) petits entrepreneurs des prêts que jamais les banques classiques n'accepteraient de consentir.

Recyclage. La modestie des moyens matériels, pour employer un euphémisme, a fait des Malgaches les rois du recyclage, dans beaucoup de domaines. Ainsi, la fabrication de petits véhicules à l'intention des touristes, avec des canettes d'alu, du fil électrique, des restes de toile cirée, etc. Très réussi, quelle inventivité et dextérité !

Dépaysements divers. Les femmes (nombreuses) au visage couvert d'un «masque» de pâte à l'allure d'argile, préparé par frottement de bois de santal sur une pierre et destiné à protéger la peau du soleil ainsi que de la chaleur du foyer. - Une partie de cricket (jusqu'où l'Empire britannique n'a-t-il pas étendu son influence?) par des enfants en haillons devant des bungalows pour gens aisés sur la plage de Nosy $\mathrm{Be}$, haut-lieu touristique. - Le vieux guitariste qui nous dit-on a eu son heure de gloire dans les clubs de la capitale et qui, dans un restaurant de province, joue les tubes français (et un peu anglo-saxons) des années 1960-1980. Avec un moment incroyable pour moi quand il chante «Etoile des neiges», histoire d'un petit Savoyard amoureux qui était omniprésente sur les ondes de la radio romande dans les années 1950 !
Solidarité. Les gens sont aimables à Madagascar; avec un sentiment marqué d'appartenance nationale en dépit de la multiplicité ethnique et de la faiblesse de la gouvernance publique. Il y a de la solidarité par delà des écarts socio-économiques. Petit exemple: alors que nous traversions une région semi désertique, notre guide-chauffeur s'est arrêté spontanément à plusieurs reprises pour remettre à des enfants et adultes au bord de la route une grande bouteille d'eau tirée de la réserve que notre $4 \times 4$ transportait pour ses passagers touristes.

Zébu. Animal domestique très répandu, il est un des «piliers» de la vie économique, sociale et culturoreligieuse, important au plan de l'élevage comme aussi signe cardinal de la richesse, légitimant en particulier le nombre d'épouses auquel on peut prétendre (par exemple en pays bara). En rapport avec des éléments relevés plus haut, on nous dit que, aussi déterminante que soit la fortune d'une personne durant son existence, le principe n'est pas d'en faire bénéficier ses enfants et proches. Après le décès, ses richesses seraient «consommées» d'une manière ou de l'autre; il se peut que les plusieurs milliers de zébus que possède un homme riche soient sacrifiés et mangés plutôt que remis à des héritiers. Un exemple encore des surprises que procurent les voyages, qui forment la jeunesse et même ceux qui sont moins jeunes. 